\author{
I.J. SOLA ${ }^{1, \infty}$ \\ V. COLLADOS ${ }^{1}$ \\ L. PLAJA ${ }^{1}$ \\ C. MÉNDEZ ${ }^{1}$ \\ J. SAN ROMÁN ${ }^{1}$ \\ C. RUIZ ${ }^{1,2}$ \\ I. ARIAS ${ }^{1}$ \\ A. VILLAMARíN ${ }^{3}$ \\ J. ATENCIA ${ }^{3}$ \\ M. QUINTANILLA ${ }^{3}$ \\ L. ROSO $^{1}$
}

\section{High power vortex generation with volume phase holograms and non-linear experiments in gases}

\author{
${ }^{1}$ Servicio Láser, Universidad de Salamanca, Pl. de la Merced s/n, 37008 Salamanca, Spain \\ ${ }^{2}$ Max Planck Institut für Physik komplexer Systeme, Nöthnitzer Straße 38, 01187 Dresden, Germany \\ ${ }^{3}$ Departamento de Física Aplicada, Universidad de Zaragoza, C/ Pedro Cerbuna, 12, 50009 Zaragoza, Spain
}

\section{Received: 4 July 2007/Revised version: 21 December 2007} Published online: 6 March 2008 • () Springer-Verlag 2008

ABSTRACT An experimental method for the production of high power optical vortices by using volume phase holographic plates is presented. Experiments in air, $\mathrm{N}_{2}$ and Ar have been performed as an example of the method's potential, observing non-linear effects and filamentation. Theoretical calculations support the conclusions.

PACS 42.40.Eq; 42.65.Hw

\section{$1 \quad$ Introduction}

Optical vortices (OVs) have been the object of intensive investigation due to their special properties concerning orbital angular momentum and spatial distribution [1]. Most of the works related to OVs have involved continuouswave (cw) and quasi-cw regimes. Recently, the generation of OVs in very short and intense light bursts has been a subject of interest. This will allow us to have access into the high-power regime for studying experimentally the behavior of non-linear vortex structures (i.e. propagation in Kerr media and filamentation).

In order to generate the OVs with femtosecond pulses, two main approaches can be found in the literature. Both techniques have been exported from the $\mathrm{cw}$ regime, where they had already been consolidated. First, spiral phase plates (SPPs) are media where the optical path increases with the azimuthal angle, yielding an output beam with a helical phase front [2]. It has been shown that it is possible to generate vortex structures at the Ti:sapphire emission wavelength with efficiency of 55\% and high damage thresholds [3]. Other materials, such as resists, offer higher efficiencies (around 80\% [4]) and still quite high damage thresholds in the case of photoresists [5]. Therefore, SPPs appear as promising candidates to high power OV generation [6]. However, a recent work establishes some limitations of the technique when very short pulses are used [7]. In fact, for spectra presenting a FWHM higher than $40 \mathrm{~nm}$, spatial chirp effects appear. Therefore, this

Fax: +34-923-2945-84, E-mail: ijsola@usal.es technique may have problems for pulses shorter than $30 \mathrm{fs}$. On the other hand, the manufacture of SPPs is expensive and demanding.

The second technique uses the so-called computer-generated holograms (CGHs), gratings presenting some dislocations in the diffraction pattern that creates the vortex. Usually they consist of an amplitude mask [8], but alternative processing of CGHs has been reported [9]. Also, dynamic CGHs have been tested using liquid-crystal media [10]. In general, the CGH technique presents lower efficiencies than those obtained with the SPPs. However, because of its simplicity of production compared to the SPP making up process, it is a quite popular technique, too. In addition, CGHs have been adapted to the femtosecond regime using $2 \mathrm{f}-2 \mathrm{f}$ set-ups, in order to compensate the spatial chirp $[11,12]$. The main limitations of CGHs to date are the low damage threshold of the materials used, preventing the generation of high-power OVs, and the very low efficiency (as an exception, CGHs consisting of bleached plates [9] and liquid crystals (LCs) [10] present higher efficiency, compared to the more common amplitude CGHs).

We propose an alternative way to generate the OVs, in order to overcome simultaneously the mentioned limitations of the CGHs. By using volume phase holograms (VPHs), we show that it is possible to generate vortices with high efficiency and power, since the damage threshold of these materials is high. The paper is structured as follows. In a first part, we present the making up process, recording the OV pattern in the VPH. The plate is used to generate OVs in the femtosecond pulse regime, characterizing the set-up performance and output. In a second part, we present observations of the non-linear and filamentation effects of high-power vortices in gases, showing the potential of the VPH experimental set-up. Theoretical simulations have been performed in order to understand the process and to establish comparison with experiments. Finally, conclusions and future perspectives are mentioned.

\section{$2 \quad$ Experimental set-up}

In order to record the VPH, the first step consists of generating an $\mathrm{OV}$ with low energy. We have recorded 


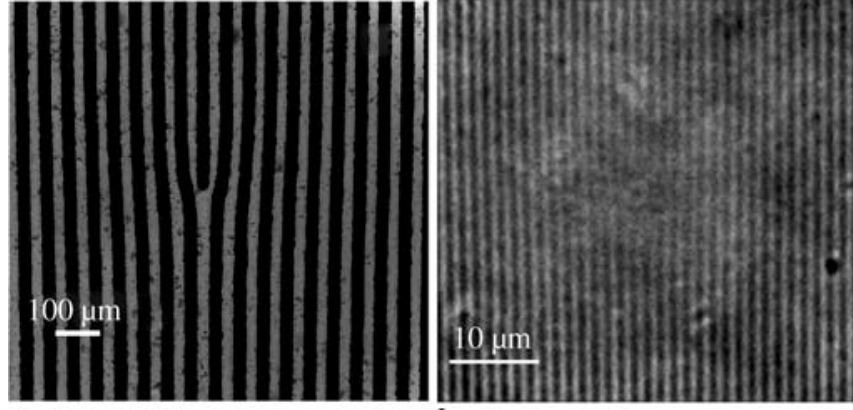

a

FIGURE 1 Microscope images. (a) Recorded plate with the micromachined $\mathrm{CGH}$ at the dislocation $(\times 5)$. (b) The singularity region of the dichromated gelatin vortex plate $(\mathrm{H} 2)(\times 100)$

by laser micromachining a $\mathrm{CGH}$ pattern on a plate, creating vortex structures in the +1 diffracted order, with topological charge $m=1$. We used a beam from a Ti:sapphire laser (110-fs-duration pulses, peak fluence of $20 \mathrm{~J} / \mathrm{cm}^{2}$ ) to print a $9 \mathrm{~mm} \times 9 \mathrm{~mm} \mathrm{CGH}$ grating. The result of this procedure is a transmission grating, presenting transparent and opaque lines (period $=55 \mu \mathrm{m}$ ). Figure 1 a shows the dislocation area of the $\mathrm{CGH}$ obtained by using a microscope (Axio Imager M1.m from Zeiss). The spatial phase dependence of the vortex (order +1 ) has been proven to be azimuthal by using the collinear interferogram between the vortex and a diverging beam as presented in [12] (see Fig. 2a). The spatial characteristics (e.g. the topological charge) can be changed just by reprogramming the micromachining in order to write the proper $\mathrm{CGH}$, and so the process can be extended easily to other topological charge vortices, or even can be generalized to other structures. The efficiency of the grating, considering the +1 diffraction order vortex, is around $9 \%$, similar to the efficiency of the usual amplitude CGHs, since several diffraction orders are generated. In contrast, the micromachined grating presents a higher damage threshold than the standard printed films or LC devices (around some tens of $\mathrm{GW} / \mathrm{cm}^{2}$ ). Experimentally, we have observed outputs of hundreds of $\mu \mathrm{J}$ (110-fs pulses), more power than that obtained typically with the common amplitude CGHs. However, the two main bottleneck points are the relatively low damage threshold (because of the absorption in the 'burned' lines) and the low efficiency.

These two points can be overcome by using volume phase holographic recordings, where the information is presented in phase modulations, not in amplitude. Volume phase holograms assure the generation of a single diffracted mode, with high efficiencies (theoretically around $100 \%$ could be possible, excluding reflection losses) depending on the experimental parameters (recording and reconstruction wavelengths, type of holographic plate, processing, etc).

In order to record the vortex VPH, we have used a typical holographic set-up. The beam of the recording laser has been split into two. One of the arms passes through the micromachined plate, generating the vortices in the different diffraction orders. The +1 order is selected, blocking the rest. The second beam is used as the reference. Both beams are directed to a holographic plate (two types were used, silver halide Slavich PFG-01 and dichromated gelatin PFG-04) forming a certain angle (depending on the plate type), needed to obtain a volume hologram for our experimental conditions.

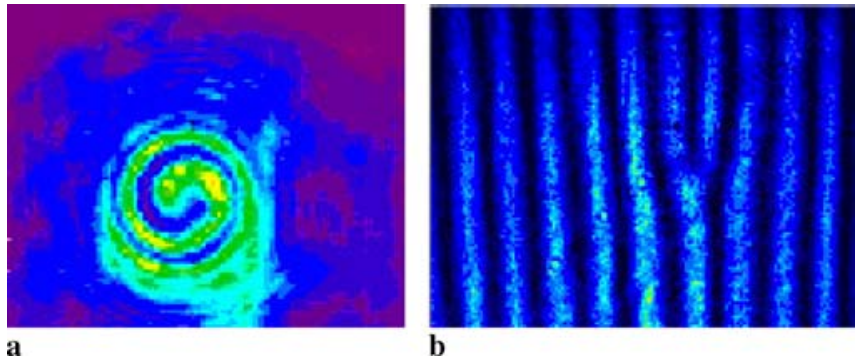

FIGURE 2 (a) Interference between the vortex generated with the micromachined CGH and a divergent beam $(\lambda=793 \mathrm{~nm})$. (b) Dislocation pattern from the VPH vortex interfering with a flat-wavefront beam $(\lambda=633 \mathrm{~nm})$

The phase nature is obtained by the development process. Figure $1 b$ shows a magnification $(\times 100)$ of the vortex region in the dichromated gelatin plate, observing a grating structure whose periodicity is 700 lines $/ \mathrm{mm}$. Note that, in this last figure, the dislocation structure can be guessed: although the vortex is located in a minimum-intensity region, and the dislocation is not visible, please observe that the part above the vortex presents a line more than that of the lower part.

A second grating, presenting the same dispersion characteristics as the VPH described above but without any dislocation, is necessary to avoid chromatism problems, as will be mentioned later. This second VPH was recorded in the same way as described previously, with the only difference that in this case both reference and object beams are plane wavefronts. Their interference creates the grating in the holographic plate. Its structure was verified by the microscope, obtaining the same period as that corresponding to the vortex VPH out of the singularity region. During the rest of this work, the non-vortex VPH will be called H1, and the vortex generator VPH will be referred to as $\mathrm{H} 2$.

In Fig. 2b, VPH vortex generation properties are checked. The non-collinear interference between a flat-wavefront beam and the vortex VPH order +1 is presented, showing a clear dislocation pattern, very similar to the original one (Fig. 1a), as expected (a cw He-Ne laser has been used for this interference).

Table 1 summarizes the main features of the two types of holographic plates used in this work. The efficiency of the plate at $\lambda=800 \mathrm{~nm}$ can be improved dramatically by choosing the proper type of holographic material and development process. Therefore, the dichromated gelatin plates seem to be the appropriate election for this wavelength, with an overall efficiency of $78 \%$, which is consistent with the results from [13]. Please note that this overall efficiency includes reflection loses. When observing the relative efficiency between the two observed orders, 0 and +1 ('efficiency ( 0 order vs. +1 order) at $793 \mathrm{~nm}$ '), since no higher diffraction orders are observed, the efficiency increases. The reason is that the main losses are due to reflection. Therefore, antireflection-coated VPHs could have even more than $90 \%$ efficiency. The recording angle between reference and object beams is also shown, and it depends on the type of plate used. Finally, the last column presents the type of recording laser.

According to their nature, the VPHs are very dispersive spectrally. A non-monochromatic beam, such as ultra-short pulses, is especially sensitive to this effect, presenting an im- 


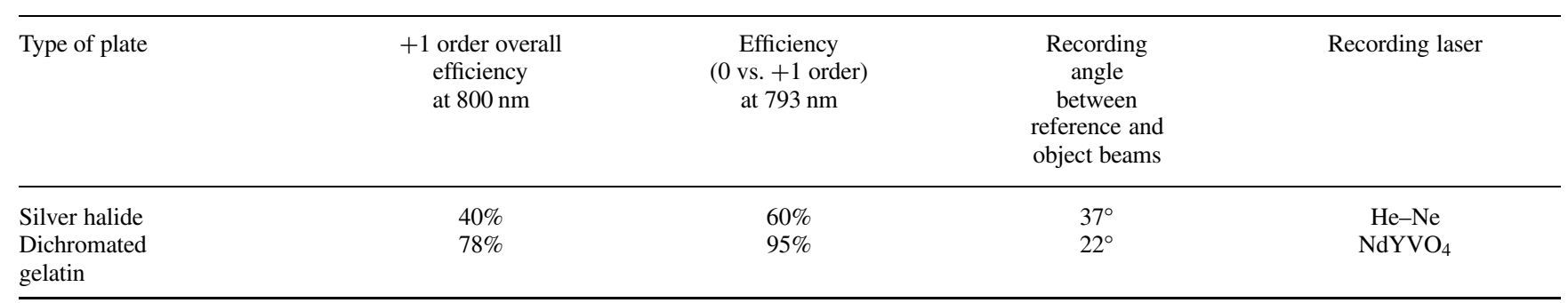

TABLE 1 Performances of the different types of VPHs

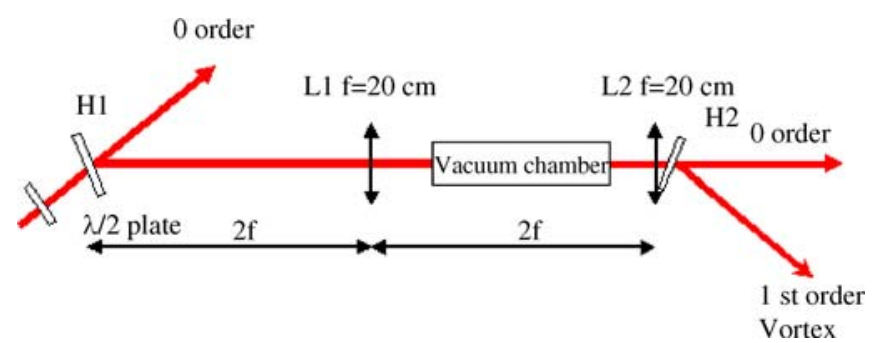

FIGURE 3 Scheme of the experimental set-up for generating the highpower vortices

portant spatial chirp in the diffracted beam. Therefore, an achromatic set-up is needed to correct this problem that could be very important in a femtosecond pulsed laser. We have implemented a set-up similar to that proposed in [12]. As shown in Fig. 3, the laser beam passes first through a half-wave plate, in order to rotate the laser polarization and optimize the holographic element response. Then, it is diffracted by the nonvortex VPH grating $\mathrm{H} 1$. A $2 \mathrm{f}-2 \mathrm{f}$ set-up (L1) images $\mathrm{H} 1$ onto $\mathrm{H} 2(f=200 \mathrm{~mm})$ with a magnification of -1 . Focalization occurs inside a vacuum cell, as quite high energy will be used and non-linear and ionization effects could appear. However, we have observed that it is a conservative precaution, at least for the powers employed in the present work, because even in air there is no important non-linear effect. Recall that the beam is very dispersed spectrally at this point, which precludes the formation of a concentrated focus. Nevertheless, for higher powers the vacuum cell may be necessary and, in general, beam stability is observed to be slightly better. Just before the vortex plate $\mathrm{H} 2$, a collimating lens L2 has been placed. We have chosen to put it before, and not after, the plate, as astigmatism of the system induced by the VPH plate is probed to be reduced dramatically.

The overall transmission of the system using silver halide VPHs is $20-15 \%$ at $793 \mathrm{~nm}$, since two VPHs are used. On the other hand, if dichromated gelatins are considered, the overall efficiency of the set-up has been observed to be around $60 \%$ at $\lambda=800 \mathrm{~nm}$, which is comparable with the SPPs' performances. Furthermore, if reflections are prevented (e.g. using antireflection coatings), the overall efficiency will soar to $80 \%$. Concerning the damage threshold, we have worked with intensities of up to $270 \mathrm{GW} / \mathrm{cm}^{2}$, without observing any damage to the holograms.

In conclusion, the VPH achromatic set-up efficiency could be similar to or even higher than those corresponding to the SPPs. Besides, they present a very broad spectral band, being especially attractive for ultra-short pulses. Vortex output power has been observed up to $130 \mathrm{GW}$, only limited by the available input power.

\section{High-power vortices in gases}

The propagation of optical vortices in Kerr media has been a subject of interest in recent years. From a fundamental point of view, the study of the dynamics of self-breaking, due to modulation instabilities, gives insight about the interplay of the vortex charge and the peak power. In the literature, several experimental works have explored the behavior of femtosecond pulsed OVs in high saturable non-linear behavior, e.g. solids [14], water [15] and gases, presenting a resonance line within the laser wavelength domain $[16,17]$. Moreover, the predicted high stability of the vortex solitons against collapse and filamentation [18] may be used for efficient and stable beam propagation in the atmosphere.

In this context, we have tested the capabilities and applications of our set-up producing OVs up to $14 \mathrm{GW}$ with the silver halide VPHs, since this power is enough to show some nonlinear effects. Due to our experimental conditions, the OVs could propagate up to $10 \mathrm{~m}$, showing no changes in the intensity distribution. But, considering calculations in [18] with similar conditions, the OVs are expected to collapse only after some hundreds of meters. In order to explore the non-linear behavior and the limits of the stability of the OVs' structure in the laboratory we loosely focus the vortex with a $f=220 \mathrm{~cm}$ convergent lens placed after the holographic plate and observe the propagation. In the linear regime in air, the vortex maintains its ring-like structure when it propagates. In the case of a 14-GW vortex, the vortex breaks up into two splinters. Figure $4 \mathrm{a}$ shows the spatial intensity distribution at $0.81 \mathrm{~m}$ after the focus for the non-linear regime. The double-splinter structure is also observed in further propagation demonstrating the generation of high-power OVs, which are able to propagate non-linearly even in atmospheric air.

In order to analyze the physical origin of these localized structures, we have performed $(2+1)$-dimensional nu-

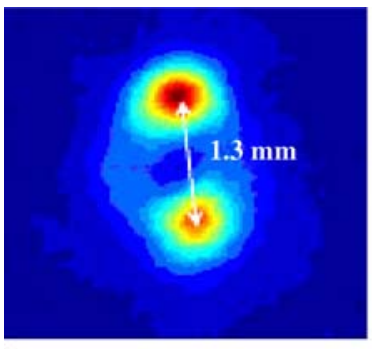

FIGURE 4 Experimental (a) and theoretical (b) split beams in air at $0.81 \mathrm{~m}$ after the focus $(f=2.2 \mathrm{~m})$, using 1.6-mJ, 110-fs pulses

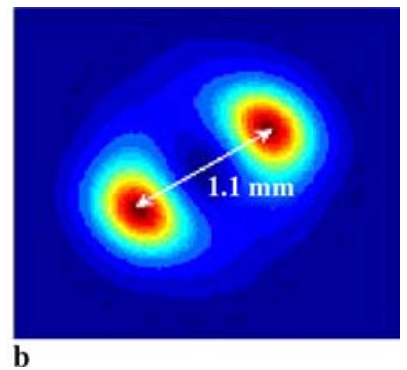

b 


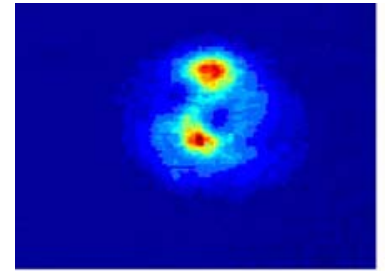

a

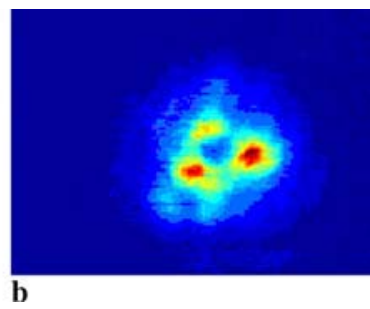

FIGURE 5 Experimental structures of filamentation at two different shots of a 14-GW vortex in $\mathrm{N}_{2}(P=1.6 \mathrm{bar})$, showing (a) two- and (b) threefilament structures

merical simulations of the propagation in air, including the Kerr non-linearity. The input intensity profile is calculated from the +1 order diffraction of a beam through a CGH, presenting the same parameters as our experimental set-up. The propagation code, solving the non-linear Schrödinger equation, is mentioned in [19]. Since our code does not include ionization, the onset of filamentation is identified as a catastrophic collapse, after which our numerical results are meaningless. For the same reason, the rise of non-singular, self-similar structures in our calculation (see Fig. 4b) can only be associated with the non-linear effect due to the Kerr effect, and not to filamentation. The good agreement of the comparison between the numerical results (Fig. 4b) and the experiments (Fig. 4a) leads us to conclude that the emerging beams are directly related to the Kerr effect.

A similar experiment as the one mentioned above was done inside a 2-m-long cell filled with $\mathrm{N}_{2}$. In these cases the intensity distributions were observed after the cell output window (around $1.33 \mathrm{~m}$ from the focus). When $P\left(\mathrm{~N}_{2}\right)=1 \mathrm{bar}$, the splitting is very similar to that observed in air. If $P\left(\mathrm{~N}_{2}\right)=$ 1.6 bar, not very far away from atmospheric pressure, the experimental behavior changes and light from multiple filamentation is clearly observed (Fig. 5). The vortex structure has been observed to collapse into two (Fig. 5a) or three (Fig. 5b) filaments in an erratic way at the same experimental conditions. In addition, numerical simulations give an indication of filamentation showing collapse near the focus.

Non-linear effects have been observed also in gases presenting a Kerr non-linear index weaker than air, for example Ar. For low Ar pressures (some hundreds of mbar), the 14-GW vortex's spatial distribution is unchanged. However, for higher pressures $(P(\mathrm{Ar})=1.9 \mathrm{bar})$, the break-up appears clearly at the cell output, splitting into two beams similarly to that shown in Fig. 4. No filamentation regime has been observed with pressures up to 2.3 bar.

These results are examples of the capabilities of the presented set-up. Using improved VPHs, such as the currently tested dichromated gelatins, leads to the generation of vortices at very high power regimes. With these high-power vortices, it is possible to have access to the non-linear regime and filamentation in air propagation, which allows us to study in a simpler and more detailed way processes such as remote filamentation, remote laser intensity breakdown spectroscopy (LIBS), etc, which can be further applied in atmospheric conditions. In addition, it is possible to perform in a simple way some modifications in the propagation, changing the angular momentum distribution.

\section{4}

\section{Conclusions}

A new system of generating optical vortices, based on recording volume phase holograms, is presented, showing high efficiencies and damage thresholds (no effects appear when irradiated with near-200-GW pulses), allowing us to produce high-power vortices. An example of the potential of this set-up has been shown in obtaining experimentally non-linear effects and break-up into two or three filaments by loosely focusing the vortices in gases $\left(\mathrm{Ar}\right.$ and $\left.\mathrm{N}_{2}\right)$. Even in air at 1 bar, non-linear effects appear, showing agreement with theoretical predictions. This opens the possibility of studying experimentally non-linear effects on vortex propagation in air, where it is easy to follow the spatial evolution or even to manipulate it. The future development of the VPHs applied to the spatial profile tailoring in the ultra-short regime, in general, and high-power ultra-short vortices, in particular, is very promising since the efficiency can be improved by using different holographic materials and procedures (e.g. in the present work, dichromated gelatin holographic plates). These high-power OVs could be employed for experimentation on filamentation, non-linear propagation even in weak Kerr media and high-harmonic generation. Moreover, the VPH technique can be generalized to the implementation of other kinds of beam shaping working at high powers.

ACKNOWLEDGEMENTS This work has been partially supported by the Spanish Ministerio de Educación y Ciencia (FEDER funds, Grant No. FIS 2006-04151). I.J. Sola acknowledges the 'Ramón y Cajal' program from the Spanish Ministerio de Educación y Ciencia.

\section{REFERENCES}

1 A. Desyatnikov, L. Torner, Y. Kivshar, Prog. Opt. 47, 1 (2005)

2 M.W. Beijersbergen, R.P.C. Coerwinkel, M. Kristensen, J.P. Woerdman, Opt. Commun. 112, 321 (1994)

3 K. Sueda, G. Miyaji, N. Miyanaga, M. Nakatsuka, Opt. Express 12, 3548 (2004)

4 W.C. Cheong, W.M. Lee, X.-C. Yuan, L.-S. Zhang, K. Dholakia, H. Wang, Appl. Phys. Lett. 85, 5785 (2004)

5 G. Witzgall, R. Vrijen, E. Yablonovitch, V. Doan, B.J. Schwartz, Opt. Lett. 23, 1745 (1998)

6 M. Fisher, C. Siders, E. Johnson, O. Andrusyak, C. Brown, M. Richardson, Proc. SPIE 6219, 621907 (2006)

7 K.J. Moh, X.-C. Yuan, D.Y. Tang, W.C. Cheong, L.S. Zhang, D.K.Y. Low, X. Peng, H.B. Niu, Z.Y. Lin, Appl. Phys. Lett. 88, 091103 (2006)

8 V.Y. Bazhenov, M.S. Soskin, M.V. Vasnetsov, J. Mod. Opt. 39, 985 (1992)

9 H. He, N.R. Heckenberg, H. Rubinsztein-Dunlop, J. Mod. Opt. 42, 217 (1995)

10 K. Crabtree, J.A. Davis, I. Moreno, Appl. Opt. 43, 1360 (2004)

11 K. Bezuhanov, A. Dreischuh, G.G. Paulus, M.G. Schätzel, H. Walther, Opt. Lett. 29, 1942 (2004)

12 I.G. Mariyenko, J. Strohaber, C.J.G.J. Uiterwaal, Opt. Express 13, 7599 (2005)

13 J.A. Arns, W.S. Colburn, S.C. Barden, Proc. SPIE 3779, 313 (1999)

14 D.V. Petrov, L. Torner, J. Martorell, R. Vilaseca, J.P. Torres, C. Cojocaru, Opt. Lett. 23, 1444 (1998)

15 L.T. Vuong, T.D. Grow, A. Ishaaya, A.L. Gaeta, G.W. Hooft, E.R. Eliel, Phys. Rev. Lett. 96, 133901 (2006)

16 V. Tikhonenko, J. Christou, B. Luther-Daves, J. Opt. Soc. Am. B 12, 2046 (1995)

17 M.S. Bigelow, P. Zerom, R.W. Boyd, Phys. Rev. Lett. 92, 083902-1 (2004)

18 A. Vinçotte, L. Bergé, Phys. Rev. Lett. 95, 193901 (2005)

19 C. Ruiz, J. San Roman, C. Mendez, V. Díaz, L. Plaja, I. Arias, L. Roso, Phys. Rev. Lett. 95, 053905 (2005) 